\title{
The WEAVE Focus Translation System: from Design to Construction
}

Manuel Canchado ${ }^{\mathrm{a}}$, Antonio Romero ${ }^{\mathrm{a}}$, Óscar Maroto ${ }^{\mathrm{a}}$, Albert Tomàs ${ }^{\mathrm{a}}$, Carlos Martín-Nuño ${ }^{\mathrm{a}}$, Joan Manel Casalta ${ }^{\mathrm{a}}$, Joaquín Prida ${ }^{\mathrm{a}}$, J. Alfonso L. Aguerrib, José Miguel Herreros ${ }^{\mathrm{b}}$, José Miguel Delgado $^{\mathrm{b}}$, José Alonso Burgal ${ }^{\mathrm{b}}$, Don Carlos Abrams ${ }^{\mathrm{c}}$, Kevin Dee ${ }^{\mathrm{c}}$, Gavin Dalton ${ }^{\mathrm{d}}$, Piercarlo Bonifacio $^{\mathrm{e}}$, Scott C. Trager ${ }^{\mathrm{f}}$, Antonella Vallenari ${ }^{\mathrm{g}}$.

${ }^{a}$ SENER Ingeniería y Sistemas, C/ Creu Casas i Sicart, 86-88, Parc de 1'Alba 08290 Cerdanyola del Vallès, Barcelona, Spain;

${ }^{\mathrm{b}}$ Instituto de Astrofísica de Canarias (IAC), Vía Lactea S/N, Tenerife, Spain;

${ }^{\mathrm{c}}$ Isaac Newton Group of Telescopes, Apartado de Correos 321, 38700 Santa Cruz de la Palma, Canary Islands, Spain;

${ }^{d}$ Dept. of Physics, University of Oxford, Keble Road, Oxford, OX1 3RH, UK;

${ }^{\text {e }}$ GEPI, L'Observatoire de Paris, 61 avenue de l'Observatoire, 75014 Paris, France;

${ }_{\mathrm{f}}^{\mathrm{f}}$ Kapteyn Institut, Rijksuniversiteit Groningen, Postbus 800, NL-9700 AV Groningen, Netherlands; INAF - Observatorio Astronomico di Padova, Vicolo Observatorio 5, 35122 - Padova, Italy.

\begin{abstract}
WEAVE is a new wide-field spectroscopy facility proposed for the prime focus of the $4.2 \mathrm{~m}$ William Herschel Telescope (WHT), placed in La Palma, Canary Islands, Spain.

To allow for the compensation of the effects of temperature-induced and gravity-induced image degradation, the WEAVE prime focus assembly will be translated along the telescope optical axis. The assembly comprises the prime focus corrector with integrated ADC, a central mount for the corrector, an instrument rotator and a twin-focal-plane fibre positioner. Translation is accomplished through the use of a set of purpose-built actuators; collectively referred to as the Focus Translation System (FTS), formed by four independently-controlled Focus Translation Units (FTUs), eight vanes connecting the FTUs to a central can, and a central can hosting WEAVE Instrument. Each FTU is capable of providing a maximum stroke of $\pm 4 \mathrm{~mm}$ with sufficient, combined force to move the five-tonne assembly with a positional accuracy of $\pm 20 \mu \mathrm{m}$ at a resolution of $5 \mu \mathrm{m}$. The coordinated movement of the four FTUs allows $\pm 3 \mathrm{~mm}$ WEAVE focus adjustment in the optical axis and $\pm 0.015^{\circ}$ tilt correction in one axis. The control of the FTS is accomplished through a PLC-based subsystem that receives positional demands from the higher-level Instrument Control System.

SENER has been responsible for designing, manufacturing and testing the FTS and the equipment required to manipulate and store the FTS together with the instrument.

This manuscript describes the final design of the FTS along with the analyses and simulations that were performed, discusses the manufacturing procedures and the results of early verification prior to integration with the telescope. The plans for mounting the whole system on the telescope are also discussed.
\end{abstract}

Keywords: FTS, FTU, PFC, translation, actuators, blades, WEAVE, WHT 
Return to the Manage Active Submissions page at http://spie.org/submissions/tasks.aspx and approve or disapprove this submission. Your manuscript will not be published without this approval. Please contact author help@spie.org with any questions or concerns.

\section{INTRODUCTION}

WEAVE is a new wide-field spectroscopy facility proposed for the prime focus of the $4.2 \mathrm{~m}$ William Herschel Telescope (WHT), placed in La Palma, Canary Islands, Spain. The instrument includes a primary focus corrector (PFC) along two degrees of freedom and a fibre positioner mechanism for 1000 fibres [1].

The PFC is in charge of maintaining the focal plane in its position along the temperature and elevation angle ranges. The temperature range induces focus misadjusting caused by the telescope structure expansion or contraction. Elevation angle changes induce tilts of the focal plane due to the huge de-centering of the Weave mass with respect to its fixation to the telescope ring and also due to the deformation of the telescope structure.

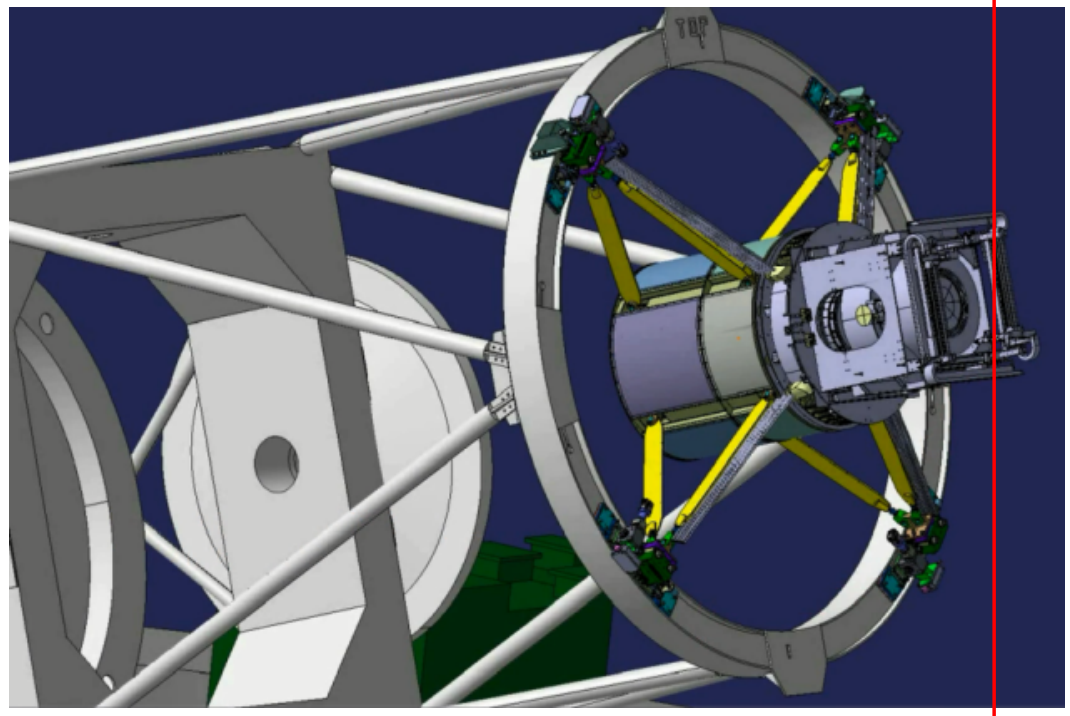

Figure 1: WEAVE installed on the William Herschel Telescope

The PFCS (Primary Focus Correction System) is composed by:

- The Focus Translation System (FTS): The FTS is composed by four Focus Translation Units (FTU) mechanisms to correct focus and tilt of the WEAVE focal plane. The four FTU are fixed to the telescope ring according the telescope disposition. Each FTU can perform a stroke of $\pm 4 \mathrm{~mm}$ (requirement is $\pm 3 \mathrm{~mm}$ to achieve the required correction for piston and tilt. It is calculated a minimum range of $\pm 2.1 \mathrm{~mm}$ for thermal excursion and $\pm 0.5 \mathrm{~mm}$ for gravitational deflection (Tilt).

- A Central Can: A beam cylindrical structure having interfaces to fix WEAVE and to fix the rotator for the fibre positioner.

- 8 Vanes: Central Can is fixed to the FTU by means of the vanes. Each pair of vanes in $\mathrm{V}$ configuration will be used for this purpose. Vanes will be preloaded conveniently to work always in tension

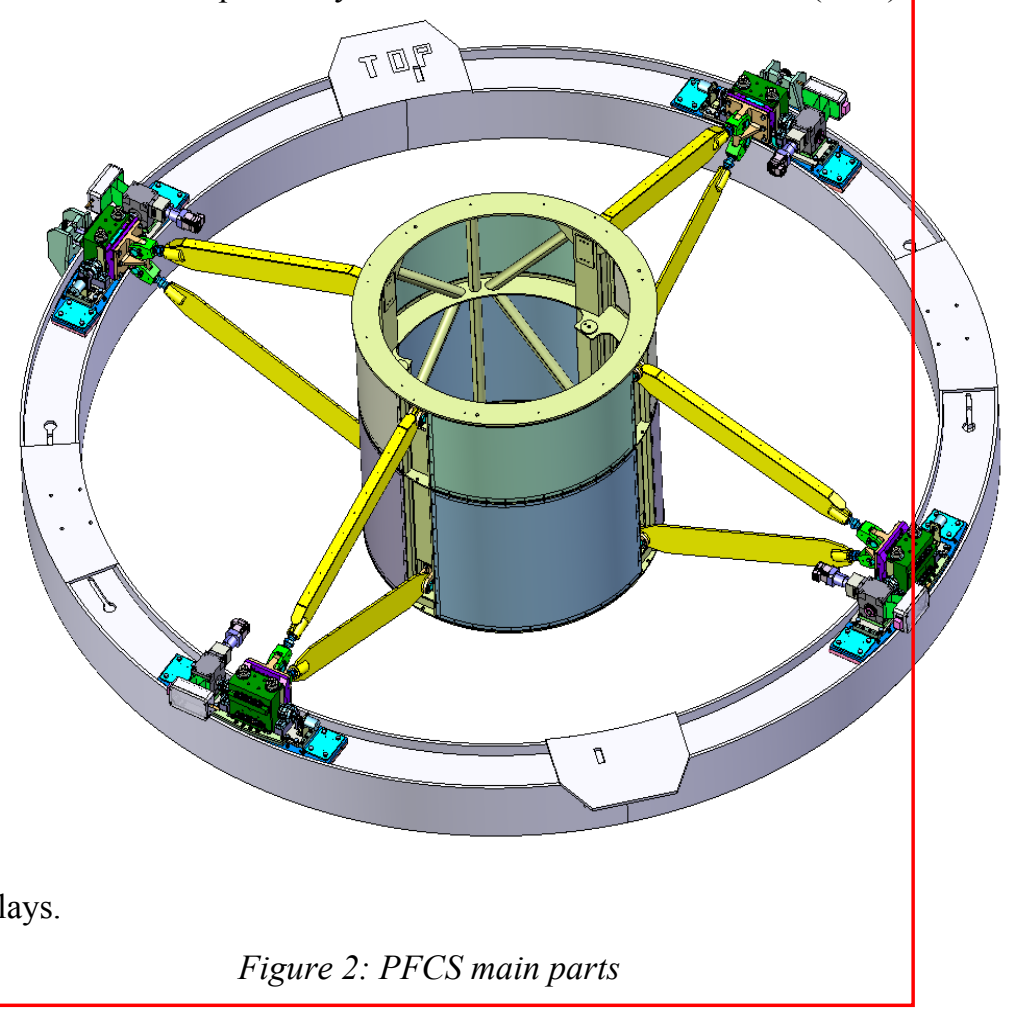
preventing the transition to compression that could induce buckling or mechanical plays 
Return to the Manage Active Submissions page at http://spie.org/submissions/tasks.aspx and approve or disapprove this submission. Your manuscript will not be published without this approval. Please contact author_help@spie.org with any questions or concerns.

- FTS Control Software (FTSCS): The focus correction is achieved with by the synchronization motion of the four FTUs. The synchronization motion is coordinated by the FTS control software, low level software implemented in a PLC. The FTSCS receives from the Observatory Control System (OCS) high level software the demanded position for each FTU to perform the focus correction. FTSCS checks the demanded position coherence and synchronize the movement of the FTS sending the individual demanded position to each FTU.

\section{- Handling Frame and Trolleys:}

A set of Auxiliary Equipment is required to perform testing operations and the operations of instrument change and maintenance. The list of those equipments is:

- Handling Frame: To allow the handling of the complete PFCS to install and removal from the telescope structure (in red in the picture below)

- Handling Trolley: Base frame on the aluminized area rails to transport the PFCS from the maintenance area (contiguous room) to the Telescope dome and vice versa.

- Working platform: structure fixed to the Handling Trolley to provide required maintenance bench for the PFCS.

- Current ring handling trolley: Structure to store the present WHT instrument.

- Tug: commercial equipment to move structures (push and pull) on the floor of the WHT facility.

- Restraining system: a set of slings, cables, chains, eyebolts, turnbuckles and additional elements required to block the telescope for instrument change supporting the huge unbalance caused by the operation.

- Test Rotator: Test bench with the capability to rotate the PFCS along the required elevation angles to tests its performances

- Mass dummies: Parts simulating envelope, mass and CoG of the WEAVE, Rotator and Fiber Positioner.
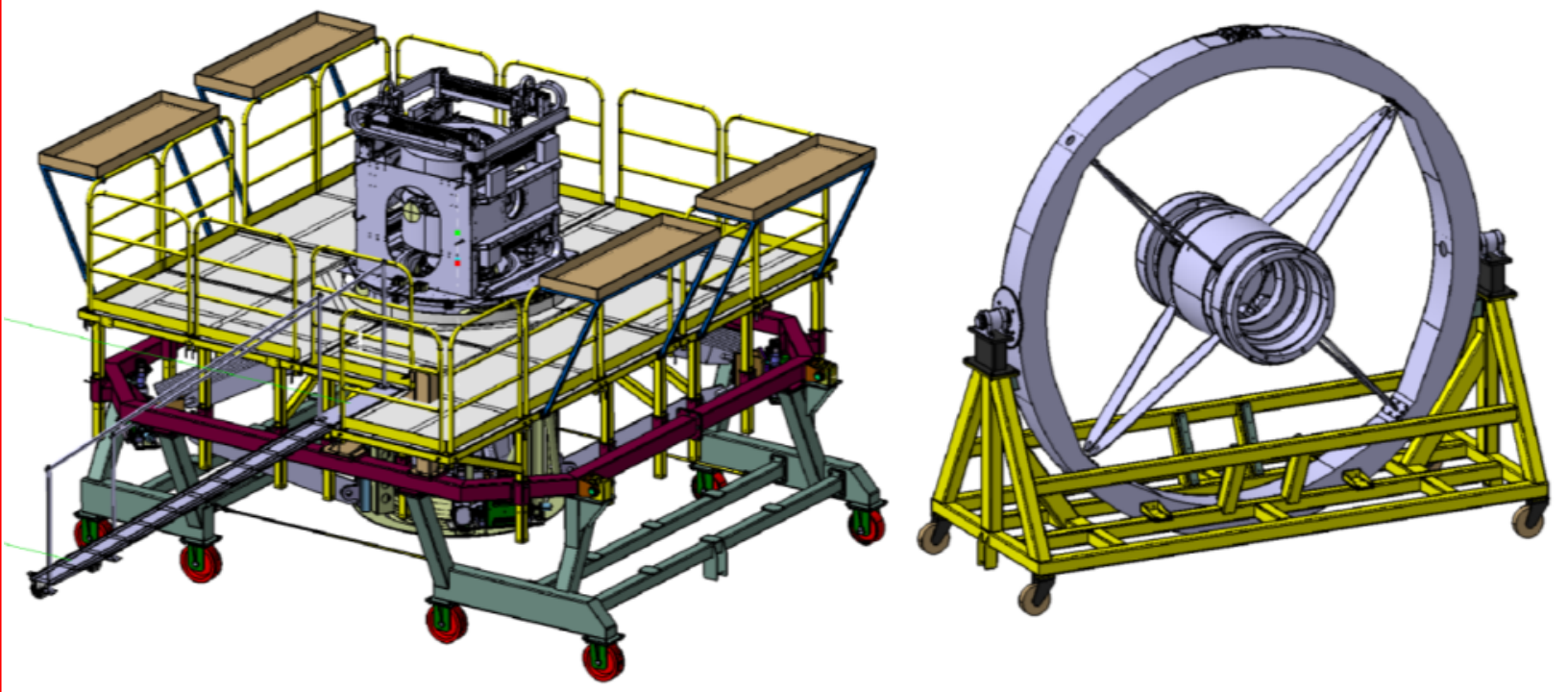

Figure 3: Handling trolleys, handling frame (red), working platform and current ring handling trolley

The main required performance specifications for PFCS are:

- FTS Equivalent force needs to translate at Zenith: $>125 \mathrm{kN}$.

- Allowable decenter of the optical axis as a function of Zenith Angle 0 to 60 degrees $+/-200 \mu \mathrm{m}$. 


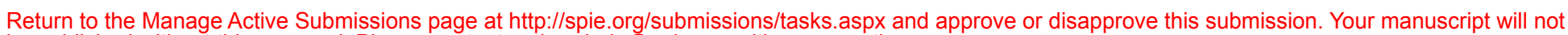
be published without this approval. Please contact author_help@spie.org with any questions or concerns.

- Focus compensation operating temperature range -5 to $25^{\circ} \mathrm{C}$.

- Range of focus linear translation must be equal or greater than $\pm 3000 \mu \mathrm{m}$.

- Accuracy must be equal or less than $\pm 20 \mu \mathrm{m}$. Goal $\pm 14 \mu \mathrm{m}$.

- Repeatability must be equal or less than $\pm 10 \mu \mathrm{m}$.

- Resolution must be less than $\pm 5 \mu \mathrm{m}$.

- $\quad$ FTS must be stable at a commanded position between $\pm 5 \mu \mathrm{m}$ until a new position is required.

- Tilt motion up to \pm 0.015 degrees.

- The time interval to obtain a new position during the operational mode must be less than 2 minutes.

- $\quad$ Speed of FTUs in Focus Position Mode must be at least $20 \mu \mathrm{m} / \mathrm{sec}$

- Speed of FTUs in Maintenance Mode must be at least 0.5 cycle $/ \mathrm{min}$

- Relative humidity from $0 \%$ to $100 \%$ (condensing).

- Operation temperature from $-5^{\circ} \mathrm{C}$ to $25^{\circ} \mathrm{C}$

- It must be robust against ingress or contamination by wind-borne Saharan dust (so called "Calima" events) $0.1-10$ um

- It must be robust against ingress or contamination by wind-borne ash from forest fires.

- The peak heat dissipated by each of the FTU or the FTS control unit must be less than 100W peak.

- Expected useful life 10 years.

- The FTU must have an MTBF of at least $10000 \mathrm{~h}$.

- The FTU must have an MTTR of less than $4 \mathrm{~h}$.

\section{WEAVE FTS DESIGN OVERVIEW}

\subsection{Focus Translation Unit (FTU) Actuator}

FTU is composed by a motor plus gearboxes and eccentric shaft (cam shaft) with the corresponding bearings to convert the rotation of the cam shaft in vertical linear motion. The whole instrument is fixed to the telescope ring by means of the Blades (made of $\mathrm{CuBe}$ ) that permits the vertical displacement (bending deformation) being stiff in plane to support the loads in-plane. The contact between the instrument and the cam shaft is assured by means of a pair of spring for each FTU. These springs will produce the needed force to withstand the huge de-centring of the instrument mass. Motor shall overcome the forces to move the mass and to deform blades and springs. 
Return to the Manage Active Submissions page at http://spie.org/submissions/tasks.aspx and approve or disapprove this submission. Your manuscript will not be published without this approval. Please contact author_help@spie.org with any questions or concerns.

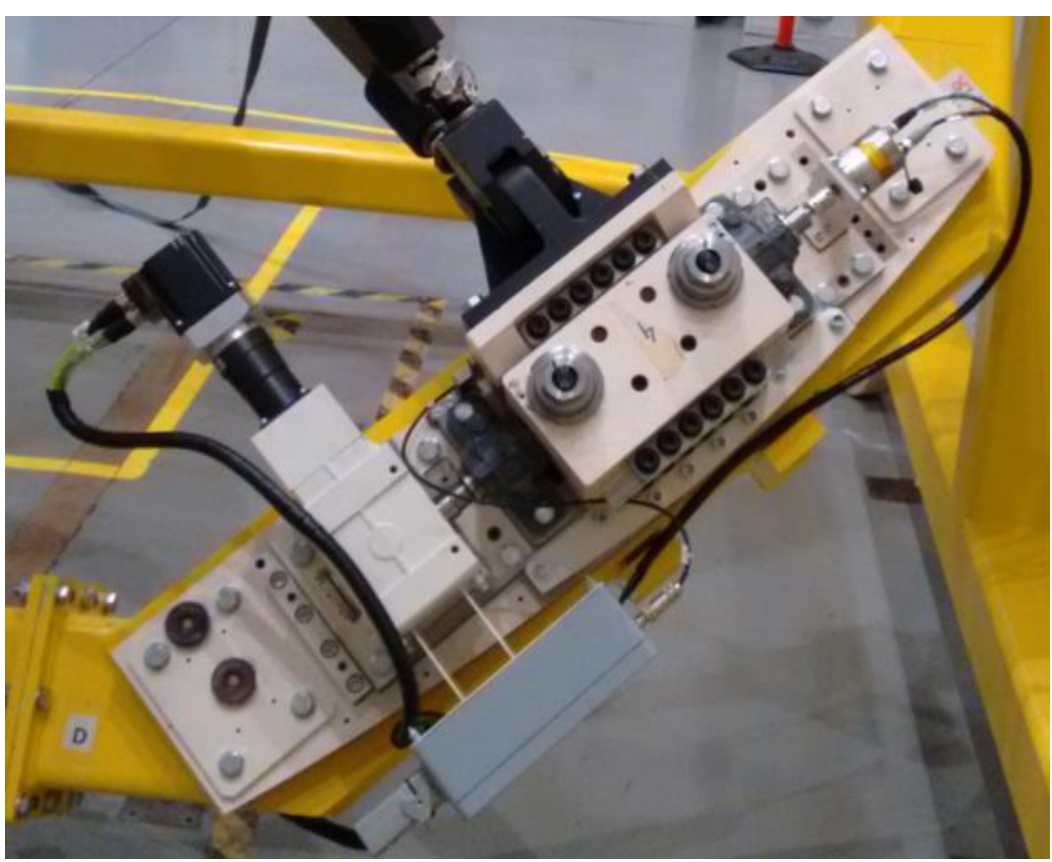

Figure 4: FTU Actuator during Acceptance tests

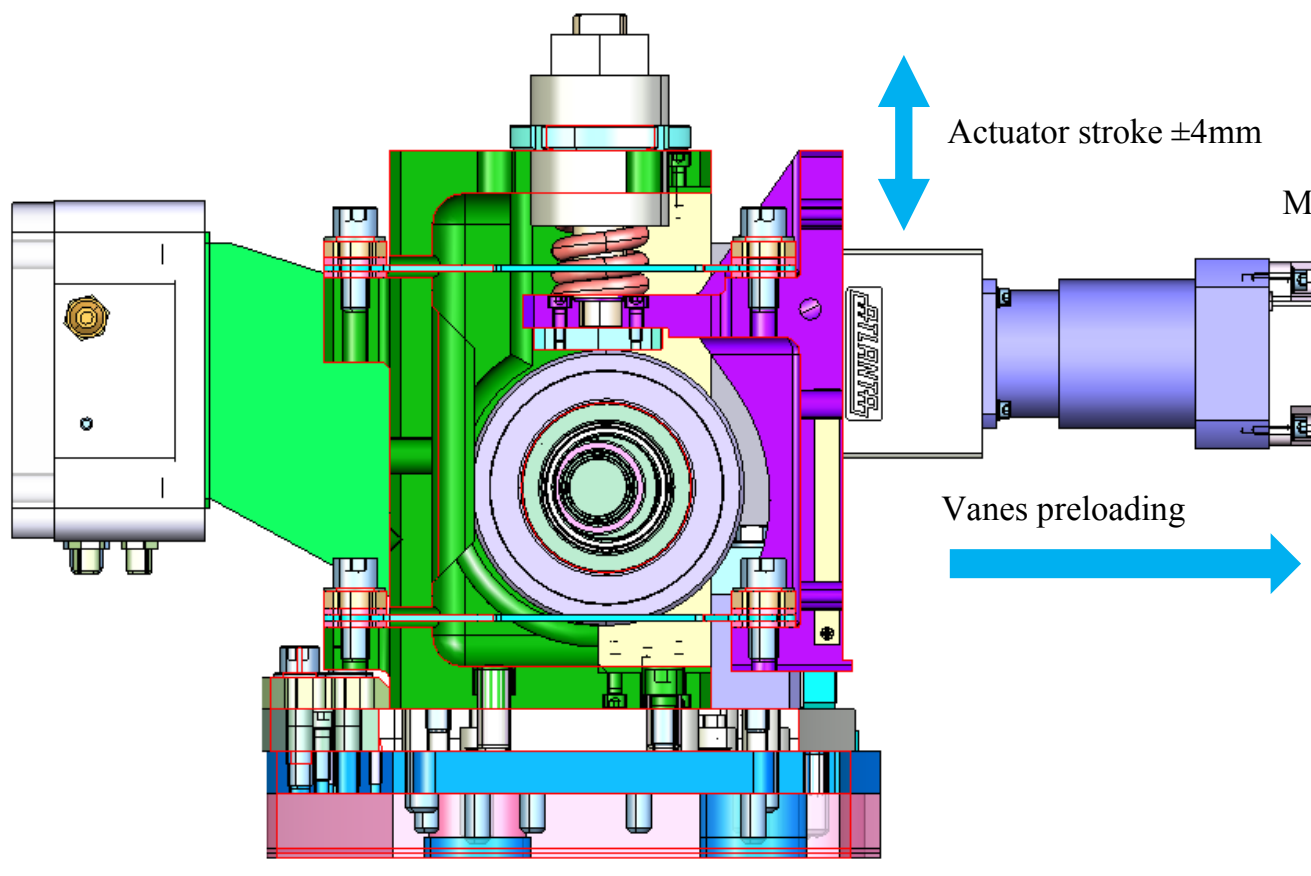

Figure 5: FTU Actuator parts

The motor through a planetary gear plus a worm gear produces the rotation of the Cam Shaft. The Cam Shaft has an Eccentric Cam which produces a linear displacement of the Stiff Finger (mobile part in purple). Cam Shaft angular rotation is measured with an Absolute Angular Encoder. Linear displacement of the Stiff Finger is measured and controlled with an incremental linear encoder. 
Return to the Manage Active Submissions page at http://spie.org/submissions/tasks.aspx and approve or disapprove this submission. Your manuscript will not be published without this approval. Please contact author_help@spie.org with any questions or concerns.

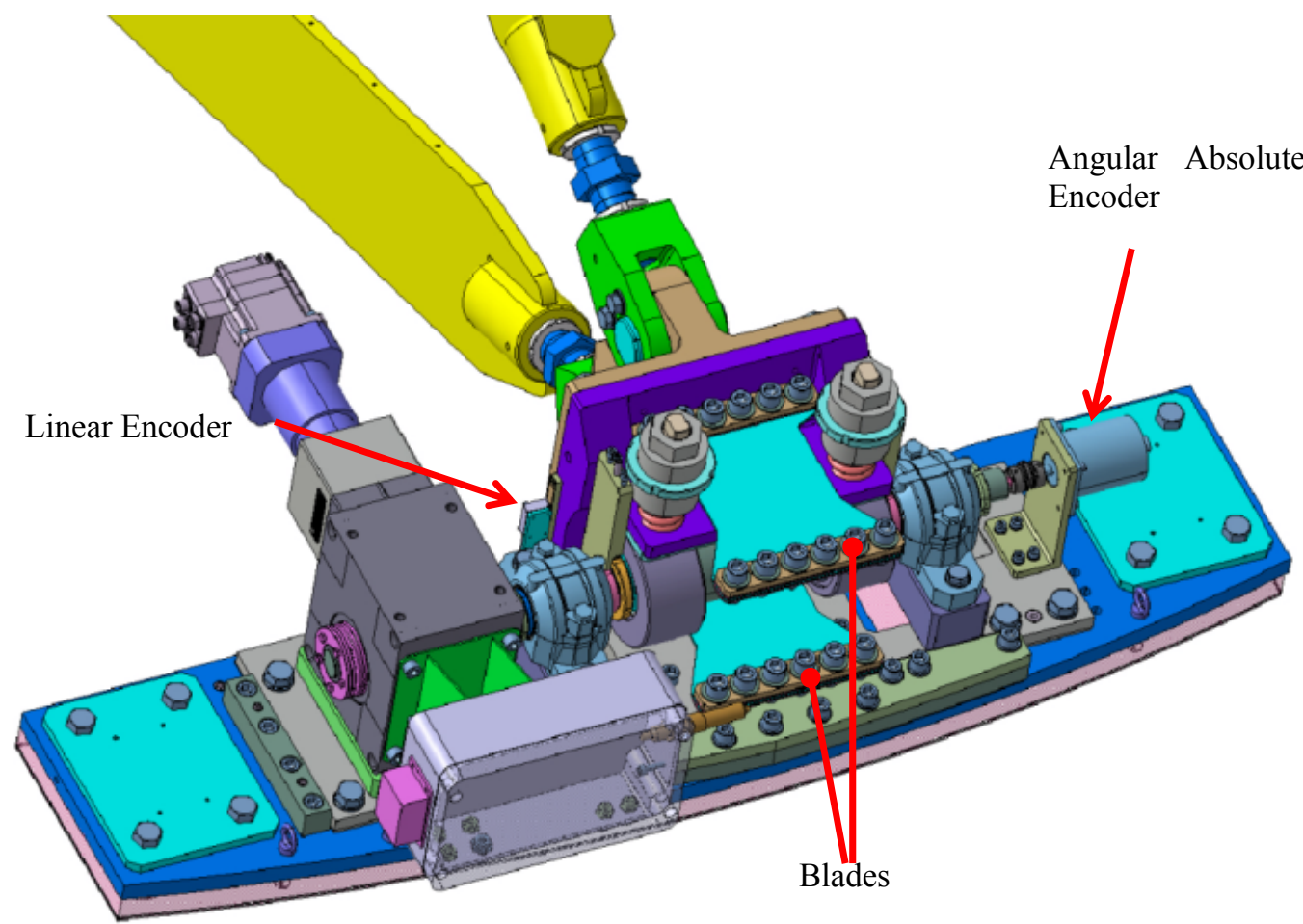

Figure 6: FTU Actuator Mechanical details

\subsection{Vanes}

Vanes are the structural parts fixing the Central Can to the FTU via the Stiff finger. Stiff finger have tensioners to adjust each vane separately. Vanes are $15 \mathrm{~mm}$ thick to be compliant with the shadow requirement. Vanes have to support instrument load and vanes preload.

Vanes have provisions (threaded holes) to fix cabling on top.

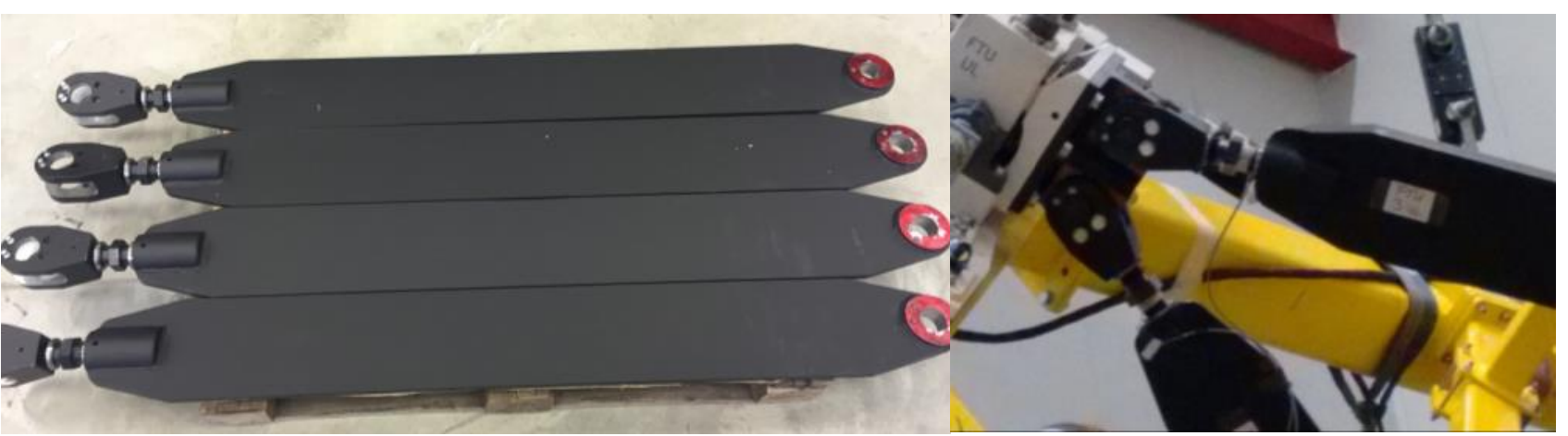

Figure 7: Vanes with tensioners and forks and detail of their attachment to the Stiff finger 
Return to the Manage Active Submissions page at http://spie.org/submissions/tasks.aspx and approve or disapprove this submission. Your manuscript will not be published without this approval. Please contact author_help@spie.org with any questions or concerns.

\subsection{Central Can}

Central Can is a welded structure providing support and interfaces for:

- the Weave (PFC) and

- the Rotator.

It's made of carbon steel, machined and painted in black.

The Central Can has the required attachments for the Vanes. The structure has provisions for an external cover bolted made in 8 sections.

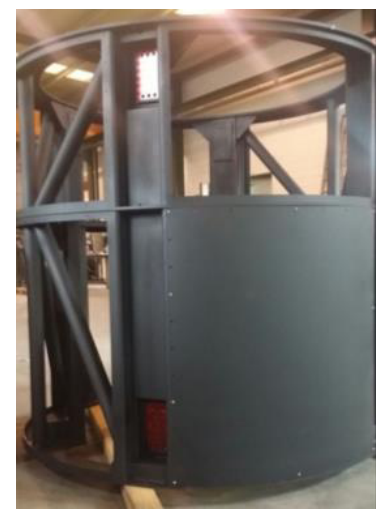

Figure 8: Central Can during manufacturing process

\subsection{FTU's electronics and electrical parts}

Position control of the Focus planes is based on the individual motion of the FTUs. To implement the control closed loop the following electrical parts were used:

- Absolute Angular Encoder: It's used to control the position of the eccentric cams in the initialization of the FTS (Home) or FTS Maintenance (FTUs cycling). Accuracy $= \pm 0.1667$ degrees, Resolution $=0.0054$ degrees

- Incremental Linear Encoder and magnetic band:. It provides the linear position for the FTU position closed control loop. Accuracy $= \pm 10 \mu \mathrm{m}$, Resolution $=0.2 \mu \mathrm{m}$, Repeatability $= \pm 1 \mu \mathrm{m}$.

- Stepper Motor and Servo Drive: Motor torque and speed characteristics have enough margin to cover the FTU operational requirements. As the Step Motor controller is embedded in the same motor enclosure has the advantage of no cables between motor and drive are needed, therefore, EMC Switching noise remains inside the motor. 24V, 9A, Max torque 3Nm, resolution $409600 \mathrm{Step} / \mathrm{rev}$.

Other advantages provided by the Step Motor controller are a selectable set of communication interfaces:

- A RS-422. It is used to interface with the linear incremental sensor

- An Ethernet IN port. It is used as a channel command to received command from FTSCS.

- An Ethernet Out port that will act as an Ethernet switch to connect the Absolute Angular Encoder.

Communication between Motor and Sensors are concentrated in the Servo Drive making the design compact and easy to be maintained.

Both Step Motor Controller and the Absolute Angular Encoder are using the Ethernet/IP protocol, then, the access of the sensor data (linear or angular) and configuration registers from the FTSCS is fully transparent using a single Ethernet cable per FTU. 
Return to the Manage Active Submissions page at http://spie.org/submissions/tasks.aspx and approve or disapprove this submission. Your manuscript will not be published without this approval. Please contact author_help@spie.org with any questions or concerns.

\subsection{Focus Translation System Control Software (FTSCS)}

FTS will be commanded from Observatory Control System (OCS) or the Engineering Interface for troubleshooting purposes. OCS generates the position demands for the FTUs to maintain the focal plane in its position along the temperature and elevation angle ranges. The FTSCS low level software was developed to ensure the synchronization of FTU's movement and check that the FTS achieve the focus plane position required by OCS.

FTSCS is implemented in embedded system platform (PLC) architecture but in fact is a distributed control due to the position control closed loop of the FTU is implemented in each FTU servo drive. PLC sends the synchronized position demands received from the Observatory Control System (OCS) to each FTUs servo drives.

FTS control software was split in two parts:

- A PLC Control SW

- A Motor Control SW residing in each FTU SMC85 Step Motor Controller

PLC Control SW performs the following task after FTS is considered initialized:

- Updating continuously the data associated to each FTU

- Processing incoming commands from OCS/ENGINT

- Checking if the demanded motion for each individual FTU are consistence in terms of the FTS motion (inside range motion and not producing mechanical stress)

- Sending individual and synchronized commands to the FTUs

- Checking during the performance of the motion that the FTUs are synchronized and the relative motions between FTUs are inside of allowed limits.

- Updating the status of the FTS (command executed)

- Acquire Eccentric CAMS angular position

Motor Control SW embedded in each FTU servo drive performs continuously:

- The acquisition of FTU linear position, Motor status and Errors

- Executing the commands required by the PLC control SW. (Move, Home, Cycling or Stop)

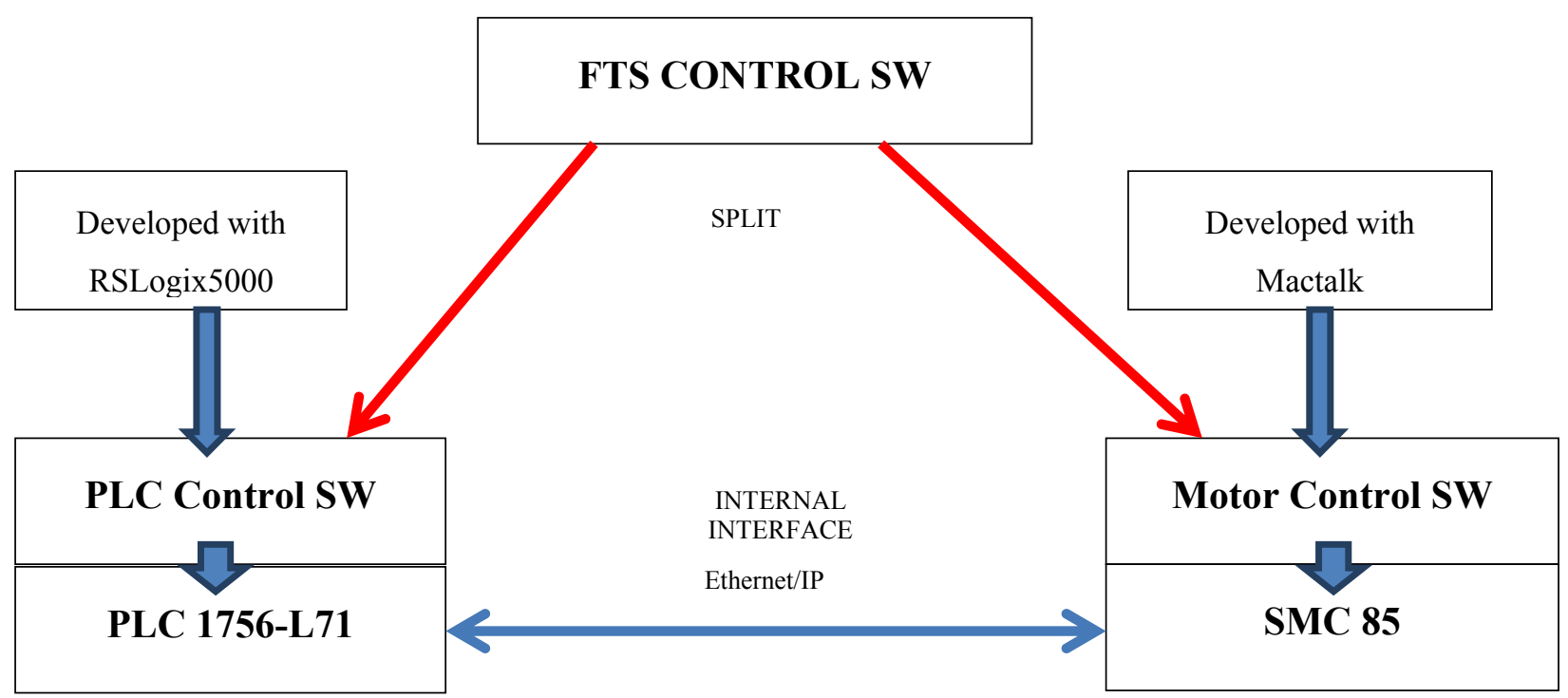

Figure 9: FTSCS architecture 
Return to the Manage Active Submissions page at http://spie.org/submissions/tasks.aspx and approve or disapprove this submission. Your manuscript will not be published without this approval. Please contact author_help@spie.org with any questions or concerns.

PLC Control SW was developed using the RSLogix 5000 development tool for Allen Bardley PLCs. The Main Routine has been developed in ladder but subroutines were implemented in structured text. Motor Control SW was developed using the MacTalk, development tool for JVL motors.

Ethernet/IP Communications protocol used by Allen Bradley PLC allows automatic detection and registers access of all the control devices involved in the control system using this interface. Therefore, PLC Control SW scans continuously the read/write assemblies of the FTUs JVL motors servo drives and encoders. It makes easy the implementation of a supervision control level in the PLC control SW in charge of analyse if new focus plane position can be achieved from the actual avoiding over tilts or mechanical stress in the FTS structure.

\section{WEAVE FTS ANALYSES RESULTS}

Geometrical, electrical, thermal and structural analyses have been carried out to verify the exposed design. The most relevant structural analysis is here summarized.

\subsection{Structural analysis}

Structural analysis is performed for the different operational cases (elevation angeles and maximum excursion in piston and tip tilt). The most stressed parts are the blades and the Support Block.

- Stresses: Structural analysis using Finite Elements Method (FEM) gives for the worst case (maximum piston of $4 \mathrm{~mm}, 1 \mathrm{~mm}$ above requirement, and $30^{\circ}$ elevation) a maximum stress on blades of $\mathbf{3 6 2}$ MPa. The admissible stress for copper beryllium is $965 \mathrm{MPa}$ to yield and thus the safety coefficient of flexures is 2.6.

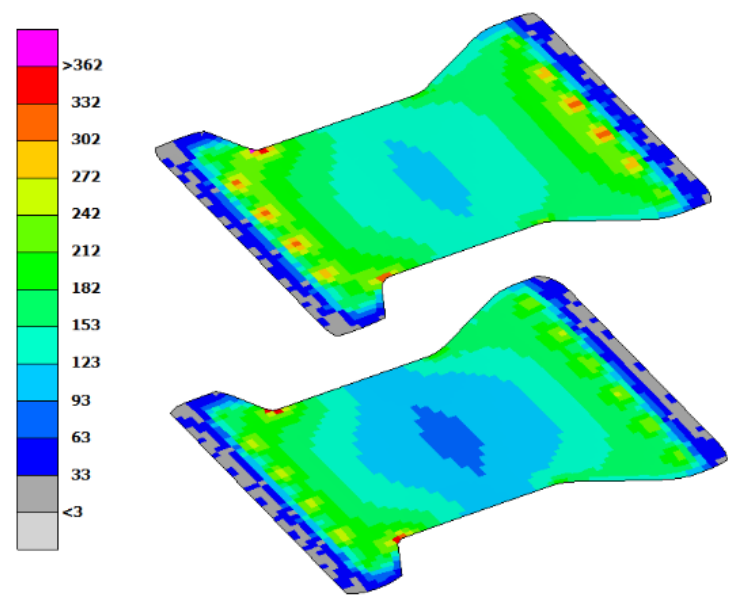

Figure 10: Worst case blade stresses (VM)

- Fatigue: The maximum operational blade stress is $305 \mathrm{MPa}$ at full nominal piston and tilt. The foreseen number of cycles is $1 \times 10^{5}$ for excursions not be always at maximum stroke. Blades have enough fatigue margin as fatigue limit for $\mathrm{CuBe}$ (reversal load) is $510 \mathrm{MPa}$ for $1 \times 10^{5}$ cycles. The fatigue margin of safety with respect to cycles is more than 100 .

Blade stresses for maximum no operational excursions $( \pm 4 \mathrm{~mm})$ are always below $362 \mathrm{MPa}$, also safe if this is repeated several times as it is below the fatigue limit for $1 \times 10^{5}$ cycles.

- Modal analysis: The first frequency of system is evaluated in $\mathbf{1 2 H z}$ (torsion) and the second is $\mathbf{1 8 H z}$ (piston). The first lateral frequency is far enough of the telescope first resonance evaluated in $10 \mathrm{~Hz}$. 


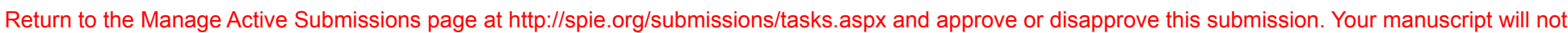
be published without this approval. Please contact author_help@spie.org with any questions or concerns.

- Actuator force: The motorization factor applied for the selection of the motor is 3 . Considering this factor the required maximum motor torque is $1.7 \mathrm{Nm}$ and the motor is capable to supply $3 \mathrm{Nm}$, so the final factor is $>3$.

- Gravitational deflection: Displacement of PFC interface and Rotator interface for the three maing altitude angles $\left(90^{\circ}, 60^{\circ}\right.$ and $\left.30^{\circ}\right)$ has been evaluated. The calculated values are:

\begin{tabular}{|l|l|l|l|l|l|}
\hline Elevation angle & $\begin{array}{l}\text { In plane } \\
\text { displacement } \\
(\mu \mathbf{m})\end{array}$ & $\begin{array}{l}\text { Displacement } \\
\text { along optical } \\
\text { axis (Z) } \\
(\mu \mathbf{m})\end{array}$ & $\begin{array}{l}\text { Tilt } \\
\text { around } \\
\text { elevation } \\
\text { axis }(\mathbf{X}) \\
(\mathbf{0})\end{array}$ & $\begin{array}{l}\text { Displacement } \\
\text { at PFC } \\
\text { interface due } \\
\text { to tilt } \\
(\boldsymbol{\mu m})\end{array}$ & Notes \\
\hline $60^{\circ}$ & -- & -- & & $\begin{array}{l}\text { Elevation for optics } \\
\text { alignment }\end{array}$ \\
\hline $\begin{array}{l}\mathbf{9 0}^{\mathbf{0}} \text { PFC } \\
\text { interface }\end{array}$ & $\mathbf{- 1 4 5}$ & $+\mathbf{6 8}$ & $\mathbf{0 . 0 0 1 6 0}$ & $+\mathbf{1 8 . 4}$ & Zenith \\
\hline $\begin{array}{l}90^{\circ} \text { Rotator } \\
\text { interface }\end{array}$ & -184 & +65 & 0.00246 & & Zenith \\
\hline $\begin{array}{l}\mathbf{3 0} \\
\text { interface PFC }\end{array}$ & $+\mathbf{1 0 4}$ & $\mathbf{- 1 9 4}$ & $\mathbf{- 0 . 0 0 0 1 9}$ & $\mathbf{- 2 . 2}$ & $\begin{array}{l}\text { Minimum elevation } \\
\text { (horizon) }\end{array}$ \\
\hline $\begin{array}{l}30^{\circ} \text { Rotator } \\
\text { interface }\end{array}$ & +134 & -176 & -0.00182 & & $\begin{array}{l}\text { Minimum elevation } \\
\text { (horizon) }\end{array}$ \\
\hline Requirement & $\pm \mathbf{2 0 0}$ & $\pm \mathbf{4 0 0}$ & - & $<\mathbf{1 5 0 . 0}$ & At focal plane \\
\hline
\end{tabular}

\section{WEAVE FTS INTEGRATION}

WEAVE FTS integration has been carried out following internal procedures used for integration of big-size hardware. A custom-made test rotator has been designed and manufactured to replicate the telescope interface flange and to allow the verification of the PFCS along the required elevation angles.

The final assembly of the PFCS on the Test Rotator has been achieved following the next summarized steps:

1- Integration of all four FTU's

2- Integration of the four FTU's on the Rotator structure (cradle)

3- Installation of the Central Can in the centre of the cradle

4- Fixation of the Vanes to the Central Can and to the Stiff Fingers

5- Install and connect all cabling

6- Load the rod tensioners of the vanes

7- Install de mass dummies inside the Central Can

8- Lift the assembly and put on the Test Rotator base.

9- Preload the vanes

The following picture shows the system once integrated with the mass dummies for the Acceptance test campaign at IAC facilities in La Laguna (Tenerife). 
Return to the Manage Active Submissions page at http://spie.org/submissions/tasks.aspx and approve or disapprove this submission. Your manuscript will not be published without this approval. Please contact author_help@spie.org with any questions or concerns.

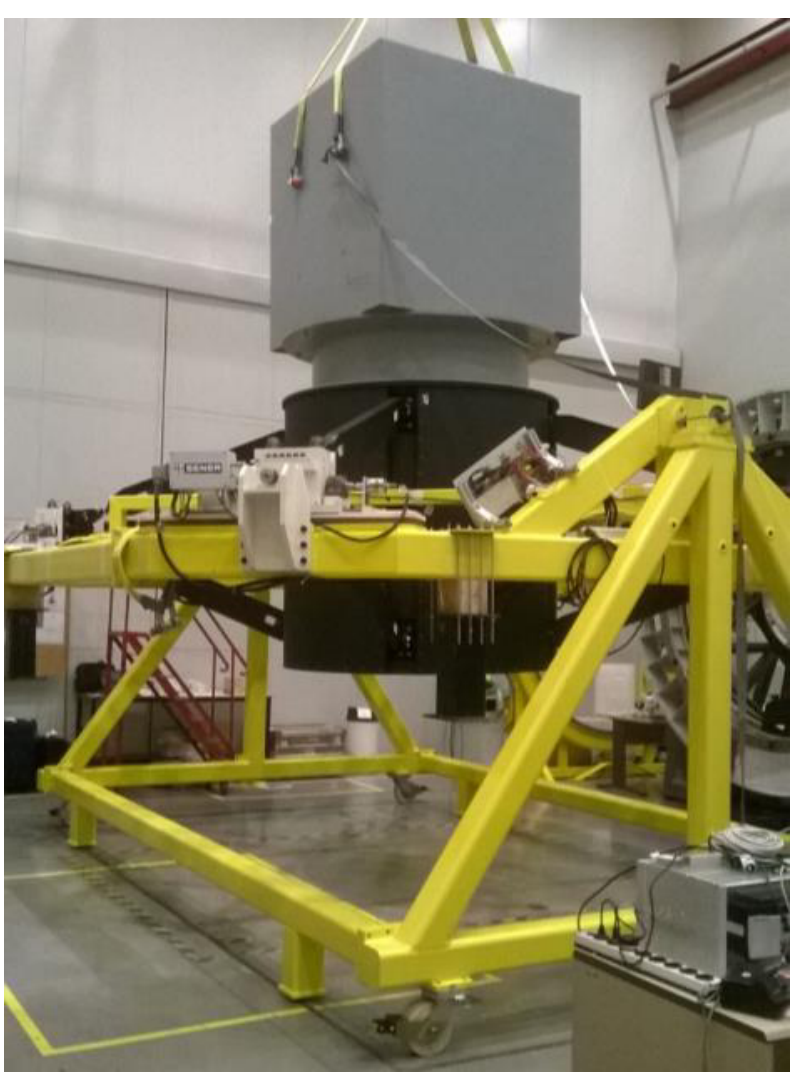

Figure 11. PFCS assembled on the Test Rotator

\section{WEAVE FTS PERFORMANCE TEST RESULTS}

\subsection{TEST SET-UP}

The Test Set-up is configured by three elements: the Rotator, the Mass Dummies and the metrology instrumentation.

The Rotator (see Figure 11) supports the WEAVE FTS and Dummies and allows performing testing at different orientations from $0^{\circ}$ to $90^{\circ}$ (discrete positions $90^{\circ}, 60^{\circ}$ and $30^{\circ}$ ) to reproduce the telescope elevation angles.

The Rotator is placed on a seismic isolation ground to minimize external perturbations during measurements.

Both the Rotator and the Dummies are adapted for the fixation of the metrology instrumentation to be used for calibration and verification of the system. Three Heidenhain linear encoders and a FARO Vantage Laser Tracker measurement system are used for these activities.

The instrumentation setup is shown in Figure 12 with the complete Test Set-up arrangement. The three linear encoders are placed pointing orthogonally to crystal surfaces on the Can to measure the focus and tilt performances and in plane arrangement to have preliminary data of deflection before testing with the Laser Tracker. 
Please verify that (1) all pages are present, (2) all figures are correct, (3) all fonts and special characters are correct, and (4) all text and figures fit within the red margin lines shown on this review document. Complete formatting information is available at http://SPIE.org/manuscripts

Return to the Manage Active Submissions page at http://spie.org/submissions/tasks.aspx and approve or disapprove this submission. Your manuscript will not be published without this approval. Please contact author_help@spie.org with any questions or concerns.

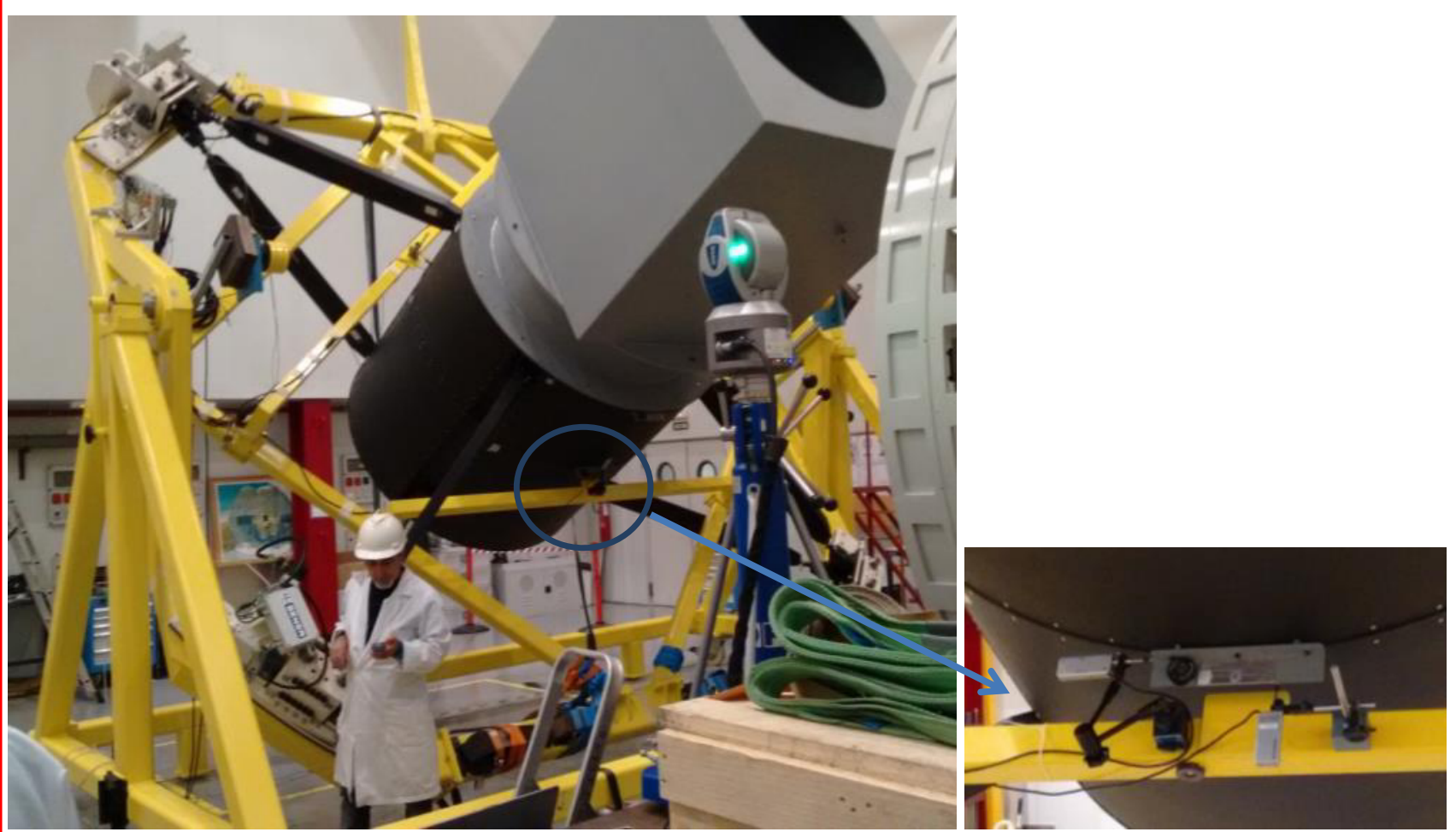

Figure 12: FARO Laser Tracker and Heidenhain length gauges for de-centring initial checks

The linear encoders are connected through an encoder data interpolating card. Data from internal sensors is sent by $\mathrm{TCP} / \mathrm{IP}$ over Ethernet at a rate of $10 \mathrm{~Hz}$ and acquired and saved jointly with external readouts. The data acquisition and reduction system is built on LabView. 


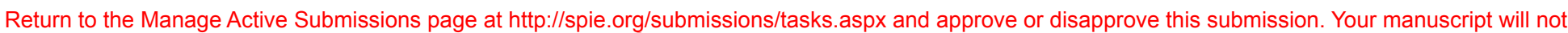
be published without this approval. Please contact author_help@spie.org with any questions or concerns.

\subsection{TEST RESULTS}

At the time of this writing the main performance results are:

- Actuator range of $\pm 3 \mathrm{~mm}$ achieved. Range measured $\pm 4 \mathrm{~mm}$ as designed. Measured with Length gauges on Central Can.

- Resolution of $5 \mu \mathrm{m}$ achieved with margins (steps of $2 \mu \mathrm{m}$ are also possible). Measured with Length gauges on Central Can.

- Repeatability of $10 \mu \mathrm{m}$ achieved. Worst measured value was $4 \mu \mathrm{m}$. Measured with Length gauges on Central Can.

- Accuracy of $20 \mu \mathrm{m}$ achieved without calibration for the range +3 to $-1.5 \mathrm{~mm}$. For the range -1.5 to $-3 \mathrm{~mm}$ requires calibration to be performed on the telescope. Measured with Length gauges on Central Can.

- Gravitational deformation of the PFC interface along the whole elevation range $30 \mu \mathrm{m}$ above the requirement of $\pm 200 \mu \mathrm{m}$ still on evaluation at the time of this writing. Measured with Laser Tracker.

Below some examples of the measured data gathered during the PFTS Acceptance Tests.

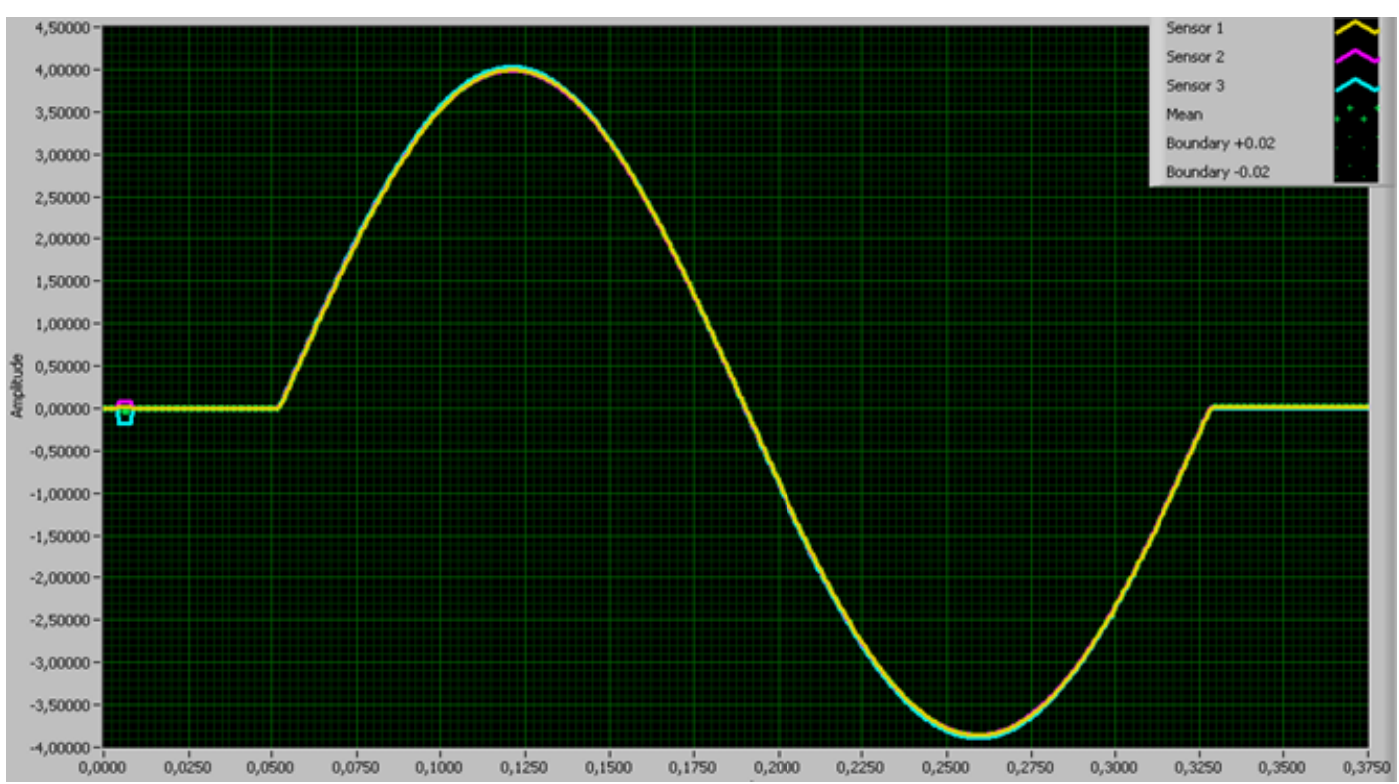

Figure 13: Range 
Please verify that (1) all pages are present, (2) all figures are correct, (3) all fonts and special characters are correct, and (4) all text and figures fit within the red margin lines shown on this review document. Complete formatting information is available at http://SPIE.org/manuscripts

Return to the Manage Active Submissions page at http://spie.org/submissions/tasks.aspx and approve or disapprove this submission. Your manuscript will not be published without this approval. Please contact author_help@spie.org with any questions or concerns.

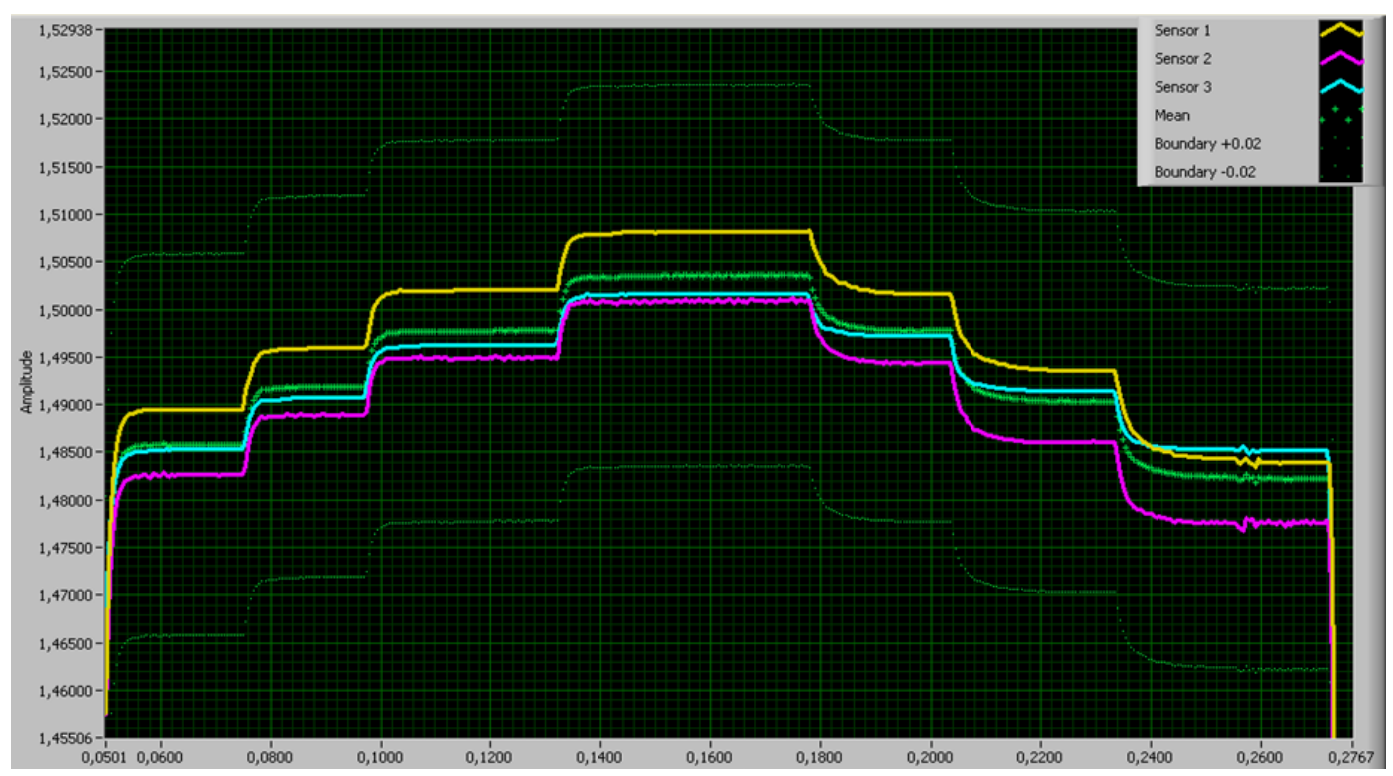

Figure 14: Accuracy without calibration and resolution. Setting at 1.485mm. Steps of $5 \mu \mathrm{m}$.

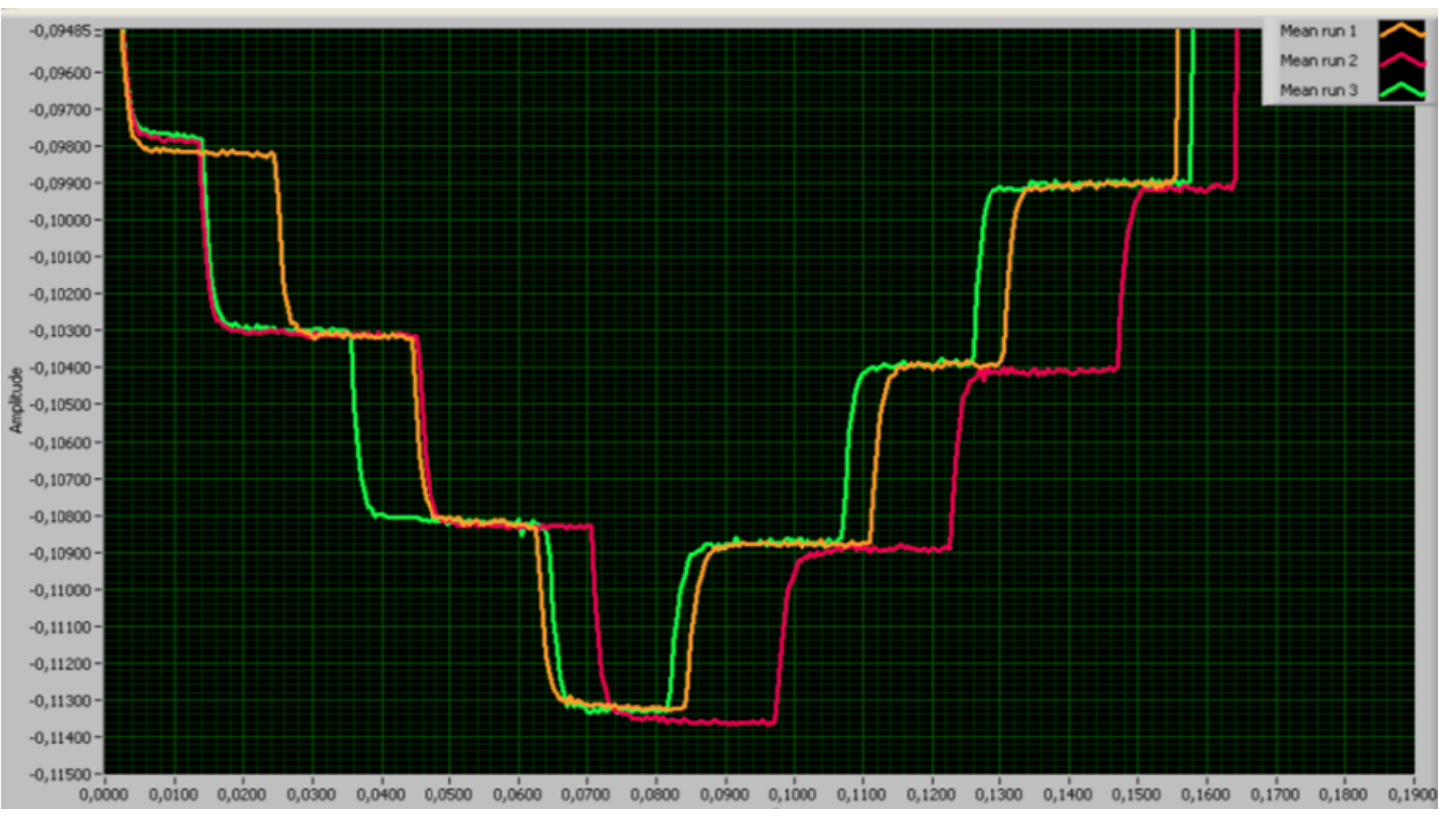

Figure 15: Repeatability of the $5 \mu \mathrm{m}$ steps. 3 runs.

\section{SUMMARY}

WEAVE FTS has been designed, manufactured, integrated and tested according to the provided specifications. The performances of the system are within the required specifications according to the test campaign. Deflection of the PFC and Rotator Interfaces along elevation angles needs to be evaluated at system level. 
Return to the Manage Active Submissions page at http://spie.org/submissions/tasks.aspx and approve or disapprove this submission. Your manuscript will not be published without this approval. Please contact author_help@spie.org with any questions or concerns.

\section{ACKNOWLEDGEMENTS}

WEAVE FTS has been designed, manufactured and tested by SENER contracted by IAC with the technical support of ING.

We would like to thank all the people involved in the project for the design of a high-precision system using off-the shelf components, but obtaining outstanding performances better than specified moreover because the schedule timeframe was very tight: all the tasks were performed in few months. Especially, thanks to the ING and IAC teams for their contributions during the project reviews and the management performed during all project phases.

\section{REFERENCES}

[1] Gavin B. Dalton et al., 'Final design and progress of WEAVE: the next generation wide-field spectroscopy facility for the William Herschel Telescope'. Paper SPIE 2016: 9908-53. 